\title{
MODELLING OF THE BREAK UP MECHANISM \\ IN GAS ATOMIZATION OF LIQUID METALS
}

\section{G.S.E. ANTIPAS}

Department of Materials

University of Oxford

Parks Road, Oxford OX1 3PH, UK

\begin{abstract}
An integrated model of the atomization of a liquid column perturbed by a flowing gas phase is proposed. The algorithm termed Surface Wave Formation (SWF) is based on the concept of the formation of sinusoidal waves travelling along the surface of the liquid. The amplitude of such instabilities can grow exponentially under specific conditions, effectively causing the detachment of liquid particles either by shearing of part of a wave crest or by detachment of a liquid body at negative amplitude nodes. The SWF model covers both primary and secondary atomization and can in principle describe the break up of any well defined liquid shape. Model predictions compare favourably with experimental data from close coupled atomization of metals. The implications of the SWF algorithm are important, since - unlike empirical correlations - it can predict the size distribution of particles inside a spray in space and atomization time. Future integration could include calculations of cooling histories of drops in flight.
\end{abstract}




\section{Introduction}

In gas atomization of melts, a liquid layer which can have the form of a column, or a sheet, is acted upon by a high velocity gas flow and is broken up into droplets. As shown in figure 1, during primary atomization, the surface of the melt is at first disturbed by a sinusoidal wave and is subsequently broken up into unstable liquid bodies, the ligaments, which in turn break down into drops. During secondary atomization, the drops may undergo further disintegration in flight.

Prediction of the size distribution of the spray produced during atomization requires an understanding of the underlying phenomena involved in the process. The majority of scientific references on this subject revolves around qualitative descriptions. For example, it is well established that increasing gas flow rates produce finer powder sizes for a fixed melt flow rate [1], [2]. An increase of the metal flow rate yields coarser particles in the spray [3], while Helium produces finer powder sizes compared to Argon and Nitrogen [4]. The particle size has also been reported to increase with increasing melt viscosity and increasing melt surface tension [5]. Macroscopic studies concentrating on particular atomization geometries have also been attempted [6], [7], [8], [9], [10]. The most popular empirical correlations on two phase jet disintegration phenomena have been reviewed by Mehrota [11], while more analytical arguments were proposed by Hinze [12], Gordon [13] and Haas [14].

\section{Figure 1.}

Although there is a large number of empirical correlations predicting mean powder sizes for certain atomization assemblies, little has been done towards a unified theoretical treatment of liquid break up. The purpose of this study is to introduce an integrated model for atomization, 
based on the surface wave formation theory, intended to describe the break up phenomena of a liquid cylindrical column perturbed by a high velocity gas flow. This model has focused on the atomization of liquid metals, but it is presented in a form that could be applicable to other liquid/gas systems.

\section{The Surface Wave Formation (SWF) Model}

The formation of a wave instability on the surface of a liquid body during gas atomization has been broadly suggested as the predominant break up mechanism of liquids in two phase flows, [15], [16], [17], [18]. In most cases, the surface wave is assumed to have a symmetric - (a) in figure 2 - or antisymmetric behaviour - (b) in figure 2 - and is thought to propagate along the direction of the relative velocity between the liquid and the gas with a finite phase velocity. The SWF theory can be extended to cover the disintegration of melts of any well defined shape interacting with high velocity gas jets, e.g. atomization of liquid sheets, as well as the break up of a stream of liquid injected into a stagnant gaseous medium.

\section{Figure 2.}

In this study, the formulation of Bradley [19] is the starting point for the derivation of the wave parameters. Bradley, based the mechanism of break up on the principle of an array of sinusoidal waves growing on the surface of a liquid column. The rate of growth of the wave amplitude, termed the growth rate, was proposed to be exponentially dependent on the wavenumber of the disturbance, the wavenumber, in turn, being defined as the inverse of the disturbance's wavelength. The wave with the largest growth rate will be the one determining the subsequent 
motion of the liquid surface, as shown in an idealized concept in figure 3. Depending on the flow conditions the surface disturbance may grow in amplitude and cause certain parts of the surface to be torn off.

\section{Figure 3.}

The SWF model initially tackles primary atomization by complementing Bradley's methodology, [19], in two critical points : 1) allowing the gravitational, $F_{g}$, to act in a direction parallel to the melt stream's surface, as can be seen in figure 3, and 2) including the effect of flow separation in the the expression of the gas pressure acting on the free liquid surface, through the use of the drag coefficient, $C_{D}$. The revised formulation yields the expression of a surface wave's growth rate, $\alpha$, in respect to its wave number, $\mathrm{k}$ :

where

the

$$
\alpha=-v k^{2}+\sqrt{v^{2} k^{4}-\frac{\sigma}{\rho_{l}} k^{3}+\frac{C_{D} \rho_{g} U_{r}^{2} k^{2}}{2 \rho_{l} \sqrt{|1-M|}}-g k}
$$

symbols are defined in table 1. Expression (1) yields positive values for the growth rate, $\alpha$, only for a limited array of wave numbers. The lower and upper boundaries of this array are respectively $\mathrm{k}_{\text {lower }}=0$ and $\mathrm{k}_{\text {upper }}$ given by equation (2)

The $\quad k_{\text {upper }}=\frac{\frac{1}{2} C_{D} \rho_{g} U_{r}^{2}}{2 \sigma \sqrt{\left|1-M^{2}\right|}}+\sqrt{\left[\frac{\frac{1}{2} C_{D} \rho_{g} U_{r}^{2}}{\rho_{l} \sqrt{\left|1-M^{2}\right|}}\right]^{2}-\frac{g \rho_{l}}{\sigma}}$

coefficient, $\mathrm{C}_{\mathrm{D}}$, was estimated by use of a protocol proposed in [20]. The amplitude of the 
surface wave will increase with respect to time, only if the growth rate is a real and positive number, or a complex number with a positive real part. As a result, amongst the possible wave numbers induced by a given set of conditions only those between $k_{\text {lower }}$ and $k_{\text {upper }}$ will favour growth. In the case of negative growth rates the surface waves will attenuate.

By means of an iterative process, the maximum growth rate, $\alpha_{\max }$, corresponding to the fastest growing wavelength, is determined. The relation between the fastest growing wavelength, $\lambda_{\max }$, and its wave number, $\mathrm{k}_{\max }$, is

After

$$
\lambda_{\max }=\frac{2 \pi}{k_{\max }}
$$

determining the fastest growing wavelength, the displacement of the liquid surface from its undisturbed position is calculated in respect with time, allowing for the fact that the instability is a progressive wave having a certain propagation velocity downstream and an exponentially growing amplitude as a function of time. At the point of initial atomization, i.e. the point the gas boundary first intercepts the surface of the liquid column, the elevation of the free liquid surface, $\mathrm{n}_{\mathrm{i}}$, is expressed as

where

$$
n_{i}=n_{o} \exp \left(a_{\max } d t\right)
$$

$\mathrm{n}_{\mathrm{o}}$ is

the

displacement of the free liquid surface at the point of initial atomization at time equal to zero and $\mathrm{dt}$ is a timestep dependent on the density of the computational grid along the surface of the column; $\mathrm{n}_{\mathrm{o}}$ is normally of the order of microns, similar to the order of the fastest growing wavelength. At any other point downstream, the amplitude of the disturbance is assumed to 
depend on the elevation of that point from the free surface in the immediately previous timestep, as expressed in equation (5)

where

$$
n_{i}=n_{o}^{*} \exp \left(a_{\max } d t\right) \cos \left(k_{\max } x\right)
$$

$\mathrm{x}$ is

the

vertical distance along the free surface of the column measured from the point of initial atomization and $\mathrm{n}_{\mathrm{o}}{ }^{*}$ is defined by the following set of conditions

2.1

$$
\text { if } n_{i}^{\text {old }}>0 \Rightarrow n_{o}^{*}=n_{i}^{\text {old }}
$$

Crest

$$
\text { if } n_{i}^{\text {old }} \leq 0 \Rightarrow n_{o}^{*}=n_{o}
$$

\section{Formation and Detachment}

If either of equations (4) and (5) produce a positive quantity of $n_{i}$, the formation of a crest on the free liquid surface at that point is assumed. In such a case the current model considers the detachment of part of that crest from the rest of the liquid body in a stripping fashion which results in the formation of a ligament. Early efforts towards the formulation of a stripping criterion were a combination of experimental observations and intuitive approaches. The common practise was to nominate a critical amplitude, normally a fraction of the fastest growing wavelength, e.g. [19]. Any part of the crest lying above this critical amplitude would be instantly sheared off the main body of the liquid. 
An alternative way to treat the problem is to apply a force balance on the crest, as shown in figure 4, an idea also proposed by Burger et al [21]. If a crest reaches an amplitude $\mathrm{n}_{\mathrm{i}}$, always measured from the undisturbed position of the free surface, the forces acting on it are

a) the drag force from the gas, $F_{g}$

b) the

$$
F_{g}=0.5 C_{D} \rho_{g} U_{r}^{2} 2 \pi\left(R_{o}+n_{i}-n_{c}\right) n_{c}
$$

liquid

surface tension, $\mathrm{F}_{\mathrm{s}}$

$$
F_{s}=2 \sigma 2 \pi\left(R_{o}+n_{i}-n_{c}\right)
$$

Stripping of part of the crest is assumed to occur if the following criterion is satisfied

The

$$
F_{g}=F_{s}
$$

system of equations (7), (8) and (9) is solved for $\mathrm{n}_{\mathrm{c}}$, which yields

In this

$$
n_{c}=\frac{2 \sigma}{0.5 C_{D} \rho_{g} U_{r}^{2}}
$$

approach, the critical amplitude is measured from the tip of the crest.

\section{Figure 4.}


Due to the cylindrical symmetry assumed in the modelling of the primary disintegration process, the detached ligament is thought to have the shape of a ring surrounding the melt column. In the present model, the volume of such a ligament, $\mathrm{V}_{1}$, is calculated by the following expression

where

$\mathrm{dx}$ is

$$
V_{l}=2 \pi\left(R_{o}+n_{i}-\frac{n_{c}}{2}\right) n_{c} d x
$$

the

average thickness of the tip being sheared off along the vertical axis. The radius of the ligament is calculated as

It is

then

$$
R_{l}=\sqrt{\frac{n_{c} d x}{\pi}}
$$

assumed that the gas inertial force and the liquid surface tension are the only forces acting on a ligament at the time of its collapse. If a part of the cylindrical ligament is sheared off, this will initially have the shape of a cylinder of radius $R_{1}$ and length $l_{c}$. At the exact time of break off the gas force, $F_{g}$ must be equal to the opposing surface tension force $F_{s}$, both acting on the globule being sheared off. This balance of forces is expressed as

where

$$
0.5 C_{D} \rho_{g} U_{r}^{2}\left(2 l_{c} \quad R_{l}\right)=\sigma 4\left(2 \pi R_{l}\right)
$$

$21_{c} R_{1}$

is the 
surface of the globule normal to the gas flow and $4\left(2 \pi R_{1}\right)$ is the total length of the new interfacial boundaries created by the shearing of the globule from the main ligament. Thus the length of the cylindrical globule is

This

$$
l_{c}=\frac{4 \pi \sigma}{0.5 C_{D} \rho_{g} U_{r}^{2}}
$$

globule is then assumed to be reformed under the effect of surface tension, into a spherical drop with diameter, $\mathrm{D}_{\mathrm{d}}$, equal to

The

$$
D_{d}=2 \sqrt[3]{\frac{3 R_{l} l_{c}}{4}}
$$

number of drops, $\mathrm{N}_{\mathrm{d}}$, produced by the initial ligament is then calculated as the ratio between the volume of the ligament, $\mathrm{V}_{\mathrm{l}}$, and the volume of the drop, $\mathrm{V}_{\mathrm{d}}$

$$
N_{d}=\frac{V_{l}}{V_{d}}=\frac{2 \pi\left(R_{o}+n_{i}-\frac{n_{c}}{2}\right) \pi R_{l}^{2}}{\frac{4}{3} \pi\left(D_{d} / 2\right)^{3}}
$$

\subsection{Detachment at Negative Amplitude Nodes}

If either of equations (4) and (5) yield a negative elevation from the free surface equal to or greater than the radius of the undisturbed melt column, a liquid globule is assumed to be 
detached from the melt at that point. The detached liquid formation is thought to have the shape of a cylinder with a radius equal to the radius of the undisturbed liquid column, $\mathrm{R}_{\mathrm{o}}$, and an average length along the vertical axis of $\lambda_{\max } / 4$. It is instantly thought to reform into a sphere under the effect of surface tension with a diameter equal to

This

mode

$$
D_{d}=\frac{1}{2} \sqrt[3]{3 R_{o}^{2} \lambda_{\max }}
$$

of

detachment is illustrated in figure 5.

\section{Figure 5.}

Finally, the numbers of the drops calculated by the SWF model have to be correlated to the actual mass flux of the liquid. The total liquid volume, $V_{t}$, injected at a mean velocity $U_{m}$ within time $t_{\max }$, is expressed as

In the

$$
V_{t}=U_{l} \pi R_{o}^{2} t_{\max }
$$

primary atomization routine, $\mathrm{t}_{\max }$ is taken to be the time needed for a crest to be formed and a ligament to be sheared from the point of initial atomization. The calculated number of drops, $\mathrm{N}_{\mathrm{i}}{ }^{*}$, for any drop size $\mathrm{i}$, is then corrected as follows 
where

$\mathrm{N}_{\mathrm{i}}^{*}$ is

$$
N_{i}^{*}=N_{i} \frac{V_{t} t^{*}}{V_{d t} t_{\max }}
$$

the

modified value of $\mathrm{N}_{\mathrm{i}}, \mathrm{t}^{*}$ is the actual atomization time to be simulated and $\mathrm{V}_{\mathrm{dt}}$ is the total volume of the calculated drop sizes within $\mathrm{t}_{\max }$. The flow chart for the SWF model is shown in figure 6.

\section{Figure 6.}

\subsection{The SWF Model for Secondary Disintegration}

The model of secondary break up is also based on the surface wave formation theory to calculate the sizes of fragments produced in a stripping fashion from the free surface of a drop. It assumes a cylindrical shape of the drop, rather than a spherical one, to facilitate computations. The volume and radius of the cylinder are taken to be the same as those of the initial spherical drop.

Each drop produced in the process of primary disintegration is assigned a point in two dimensions, defined by the axial and radial co-ordinates of its centre. After the new velocity components are established, the drop is transported to a new position inside the flow, where the relative velocity between the drop and the gas will give rise to a new set of sinusoidal wave parameters, as described in [22]. The maximum growth rate and corresponding wavelength for the new set of flow conditions are determined by equations (1) and (2), respectively. 


\section{SWF model comparison with Experimental Data}

The SWF algorithm presented here was tested against published experimental data from atomisation runs under various conditions.

Figure 7 shows the behaviour of the D32 particle size in respect to atomization pressure, for an Al-10\% M alloy atomized by $\mathrm{He}$ in a close coupled atomizer as referenced by Antipas [22]. The gas to melt mass flux ratio was kept equal to one for the whole range of injection pressures. The initial diameter of the melt column was 3mm. The SWF-predicted D32 size is within a range of \pm 10 um of the measured value and reaches a constant value of approx. $10 \mathrm{~mm}$ for pressures over 350psi (2.41mpa).

\section{Figure 7.}

Figure 8 is a comparison between SWF and experimental data from a free fall atomizer. The data is from atomization of an Al 2014 alloy by N2 as presented by Kim et al [2] and Akhlaghi et al [23]. The experimental mass median diameter and the predicted SWF D32 and D50 sizes are indeed close. Both figures 1 and 2 verify the trend of reducing drop sizes with increasing gas pressure, also observed by See et al [1] and Kim et al [2].

Figure 8. 
The most pronounced weakness of most empirical expressions tackling particle size distributions is the lack of spatial resolution. Effects such as the atomizer geometry are not taken into account and information is only given on the overall mean particle size, i.e. the most popular particle diameter found if the spray is considered as a whole. The SWF algorithm can provide such spatial resolution and tested against radial measurements of drop sizes presented in [2] and [23] shows tight agreement with experimental data, as shown in figure 9.

\section{Figure 9.}

Figure 10 is a comparison of the D50 size for a number of Al alloys, with different solute elements, atomized by $\mathrm{He}$ in a close coupled atomizer described in [22], where experimental analysis considered the sub-200mm powder fraction. The effect of increasing gas to melt mass flux ratio is similar to increasing gas injection pressure at a constant melt flowrate. As figure 10 indicates, that is the case in both the experimental and the theoretical trends. The SWFpredicted sizes show good agreement with the experimental values. Both theory and experiment also seem to suggest that there is a dramatic decrease in drop sizes for gas to melt mass flux ratios of 3 or higher.

Figure 10.

A similar comparison was made for a number of Fe alloys atomized by Nitrogen as presented in [22] and shown in figure 11. The drops in this case, both experimentally observed and predicted, are notably larger compared to those of figure 10, a result of the denser and more viscous nature of the Fe alloys as opposed to Al, as well as the effect of N2 which produces 
coarser fragments in comparison to He. Furthermore, the range of flux ratios treated in the case of the Fe alloys is markedly narrower in comparison to that of the $\mathrm{Al}$ alloys.

Figure 11.

\section{Conclusions}

An integrated model termed SWF after "Surface Wave Formation" for primary and secondary atomization of a liquid column perturbed by a gas flow was presented. The model was based on thermodynamic first principles and facilitates insight in terms of the spatial distribution of particles in two-phase flows. Predictions of the model were compared against experimental data for a range of metallic alloys and atomization conditions, showing good overall agreement. Important engineering implications of this model include the ability to assess the spatial distribution of particles inside a two phase turbulent spray.

\section{Reference List}

[1] See J. and Johnston G., Powder Technology, vol.21, p.119-133 (1978)

[2] Kim M. and Jones H., Proc. 4th International Conference on Rapidly Quenched Metals, p.85-88 (Japan 1981)

[3] Ingebo R., Journal of Propulsion and Power, vol.4, no.5, p.406-411 (1988)

[4] Ünal A., Materials Science and Technology, vol.3, p.1029-1039 (1987) 
[5] Snyder H., Senser D. and Lefebvre A., Transactions of the ASME, vol.111, p.342-347 (1989)

[6] Hiroyasu H., Shimizu M. and Arai M., ICLASS-82 p.69-74 (1982)

[7] Pai B., Nijaguna B., ICLASS-82, p.29-35 (1982)

[8] Reitz R., Atomization and Spray Technology, vol.3, p.309-337 (1987)

[9] Arai M., Shimizu M. and Hiroyasu H., ICLASS-88, p.177-184 (1988)

[10] Zanelli S., ICLASS-88, p.1-14 (1988)

[11] Mehrota S., Powder Metallurgy International, vol.13, no.2, p.80-84 and no.3, p.132$135(1988)$

[12] Hinze J., AIChE Journal, vol.1, no.3, p.289-295 (1955)

[13] Gordon G., Journal of Applied Physics, vol.30, no.11, p.1759-1761 (1959)

[14] Haas F., AIChE Journal, vol.10, no.6, p.920-924 (1964)

[15] Taylor G., Proc. Roy. Soc., A 201, p.192-196 (1950)

[16] Squirre H., British Journal of Applied Physics, vol.4, p.167-169 (1953)

[17] Keller J. and Kolodner I., Journal of Applied Physics, vol.25, no.7, p.918-291 (1954)

[18] Dombrowski N. and Johns W., Chemical Engineering Science, vol.18, p.203-214 (1963)

[19] Bradley D., J.Phys.D : Appl.Phys., vol.6, p.1724-1736 and 2267-2272 (1973)

[20] Szekely J. "Fluid Flow Phenomena in Metals Processing", p.256-259, 357- 361, Academic Press, New York (1979)

[21] Burger M., Berg E., Cho S. and Schatz A., Powder Metallurgy International, vol.24, no.1, p.32-38 (1992)

[22] Antipas G., PhD Thesis, University of Surrey, UK (1995)

[23] Akhlaghi F., Beech J. and Jones H., 1st Internatinal Conference on Spray Forming, Section 3, Paper 12 (1990) 


\section{$\underline{\text { Figure Captions }}$}

Figure 1. The principle of atomization

Figure 2. Symmetric, a, and antisymmetric, b, modes of surface oscillation.

Figure 3. The formation of a wave instability on a liquid column

Figure 4. The concept of the critical amplitude.

Figure 5. Detachment of a drop from the main body of the liquid column.

Figure 6. The flow chart of the SWF algorithm.

Figure 7. Experimental [22] and calculated D32 sizes for an Al-10wt\% Mg alloy atomized by He.

Figure 8. Experimental [23] and calculated mean particle diameter values for an Al 2014 alloy atomized by $\mathrm{N} 2$.

Figure 9. Radial variation of experimental [23] and calculated D32 values for an Al 2014 alloy atomized by $\mathrm{N} 2$.

Figure 10. Experimental [22] and calculated D50 values for a range of Al alloys with different solute elements atomized by He.

Figure 11. Comparison of experimental [22] and calculated D50 values for a range of different Fe alloys atomized by N2.

Table 1. List of symbols 


\section{Table 1}

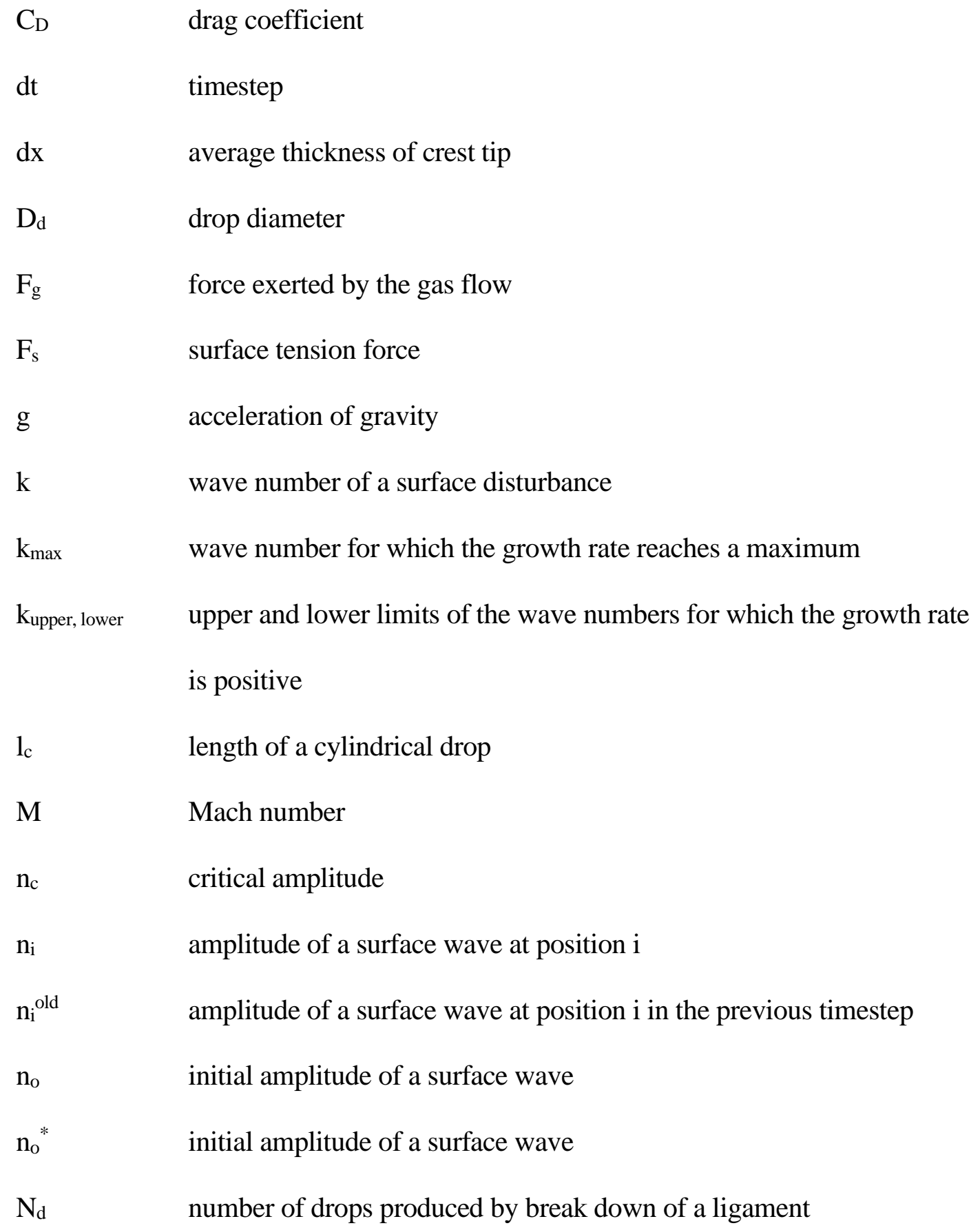




\begin{tabular}{|c|c|}
\hline $\mathrm{N}_{\mathrm{i}}, \mathrm{N}_{\mathrm{i}}^{*}$ & number of particles produced in primary break up \\
\hline $\mathrm{N}_{\mathrm{re}}$ & Reynolds number : $\rho_{\mathrm{g}} \mathrm{U}_{\mathrm{r}} \mathrm{n}_{\mathrm{i}} / \mu_{\mathrm{g}}$ \\
\hline $\mathrm{R}_{1}$ & radius of the cross section of a cylindrical ligament \\
\hline $\mathrm{R}_{\mathrm{o}}$ & radius of an undisturbed cylindrical melt column or an undisturbed drop \\
\hline $\mathrm{t}^{*}$ & real atomization time \\
\hline$t_{\max }$ & theoretical atomization time \\
\hline $\mathrm{U}_{\mathrm{m}}$ & mean initial melt exit velocity \\
\hline $\mathrm{U}_{\mathrm{r}}$ & relative velocity between the liquid and the gas phase \\
\hline $\mathrm{V}_{\mathrm{d}}$ & volume of a drop \\
\hline $\mathrm{V}_{\mathrm{dt}}$ & total calculated number of drops within $t_{\max }$ \\
\hline $\mathrm{V}_{1}$ & volume of a cylindrical ligament \\
\hline $\mathrm{V}_{\mathrm{t}}$ & total liquid volume injected \\
\hline$\alpha$ & growth rate of the disturbance \\
\hline$\alpha_{\max }$ & maximum growth rate of the disturbance \\
\hline$\lambda$ & wavelength of the disturbance \\
\hline$\lambda_{\max }$ & fastest growing wavelength \\
\hline$\mu_{\mathrm{g}}$ & gas dynamic viscosity \\
\hline$v$ & liquid kinematic viscosity \\
\hline$\rho_{\mathrm{g}}$ & gas density \\
\hline$\rho_{1}$ & liquid density \\
\hline$\sigma$ & liquid surface tension \\
\hline$\chi$ & co-ordinate along the direction of propagation for a surface wave \\
\hline
\end{tabular}




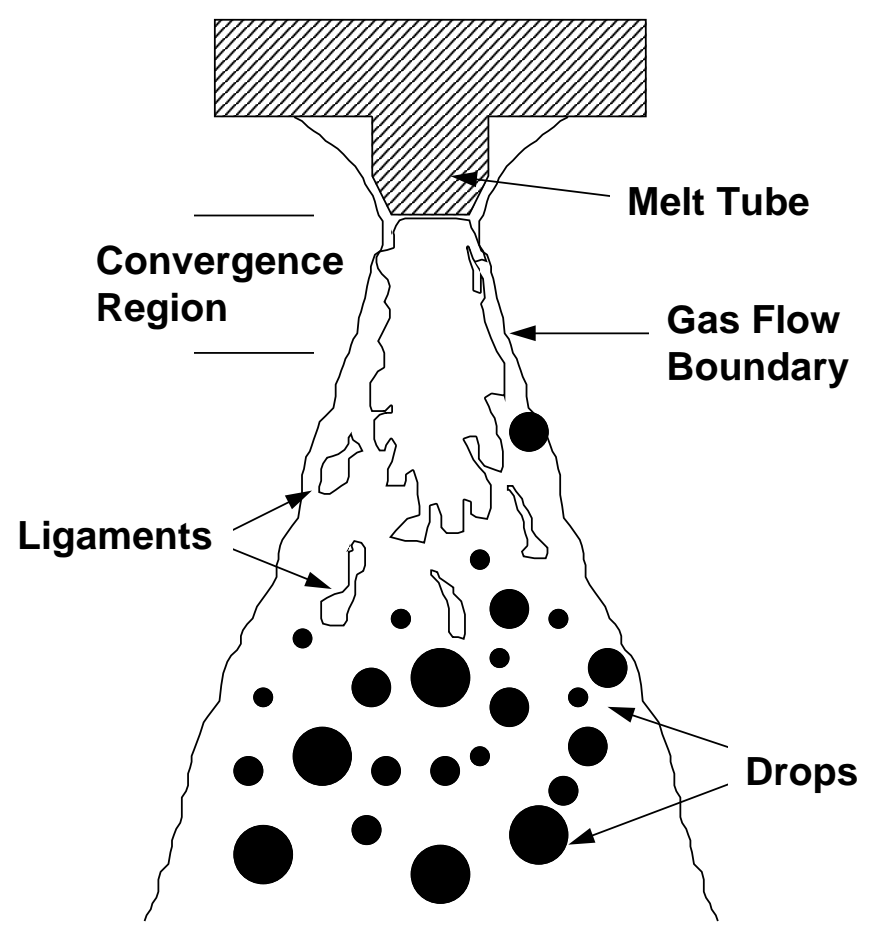

Figure 1.

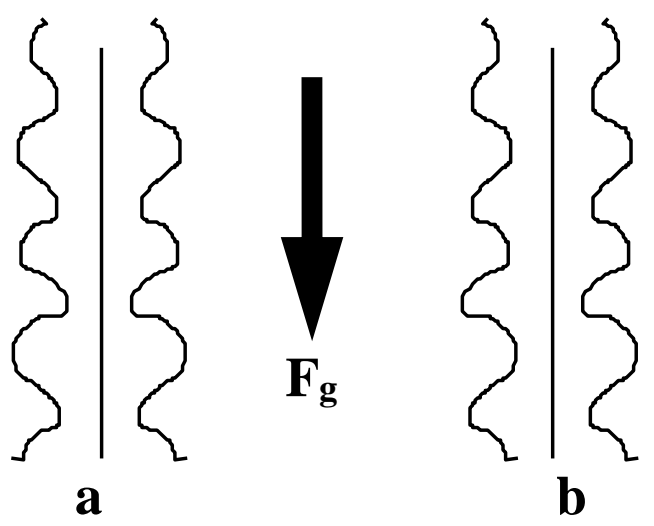

Figure 2. 


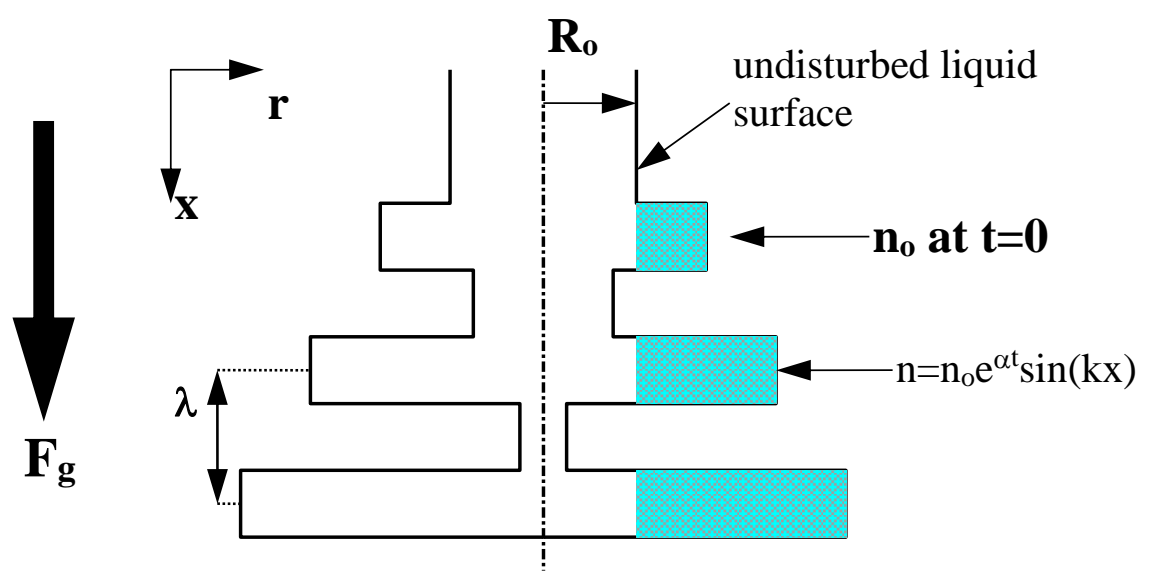

Figure 3.

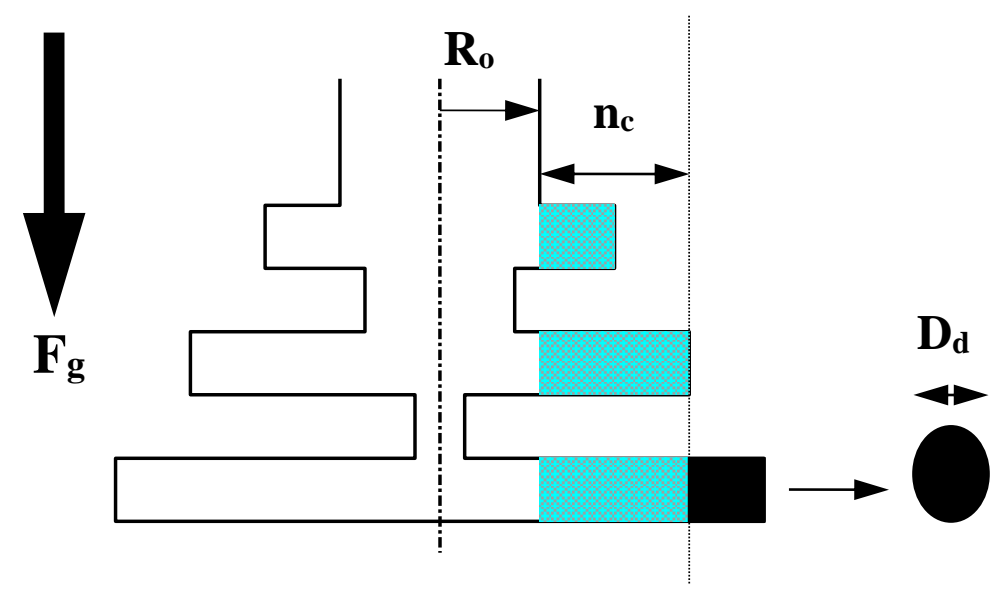

Figure 4.

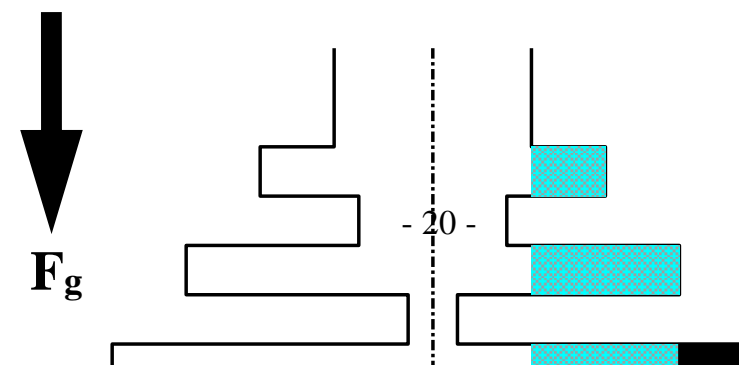


Figure 5.

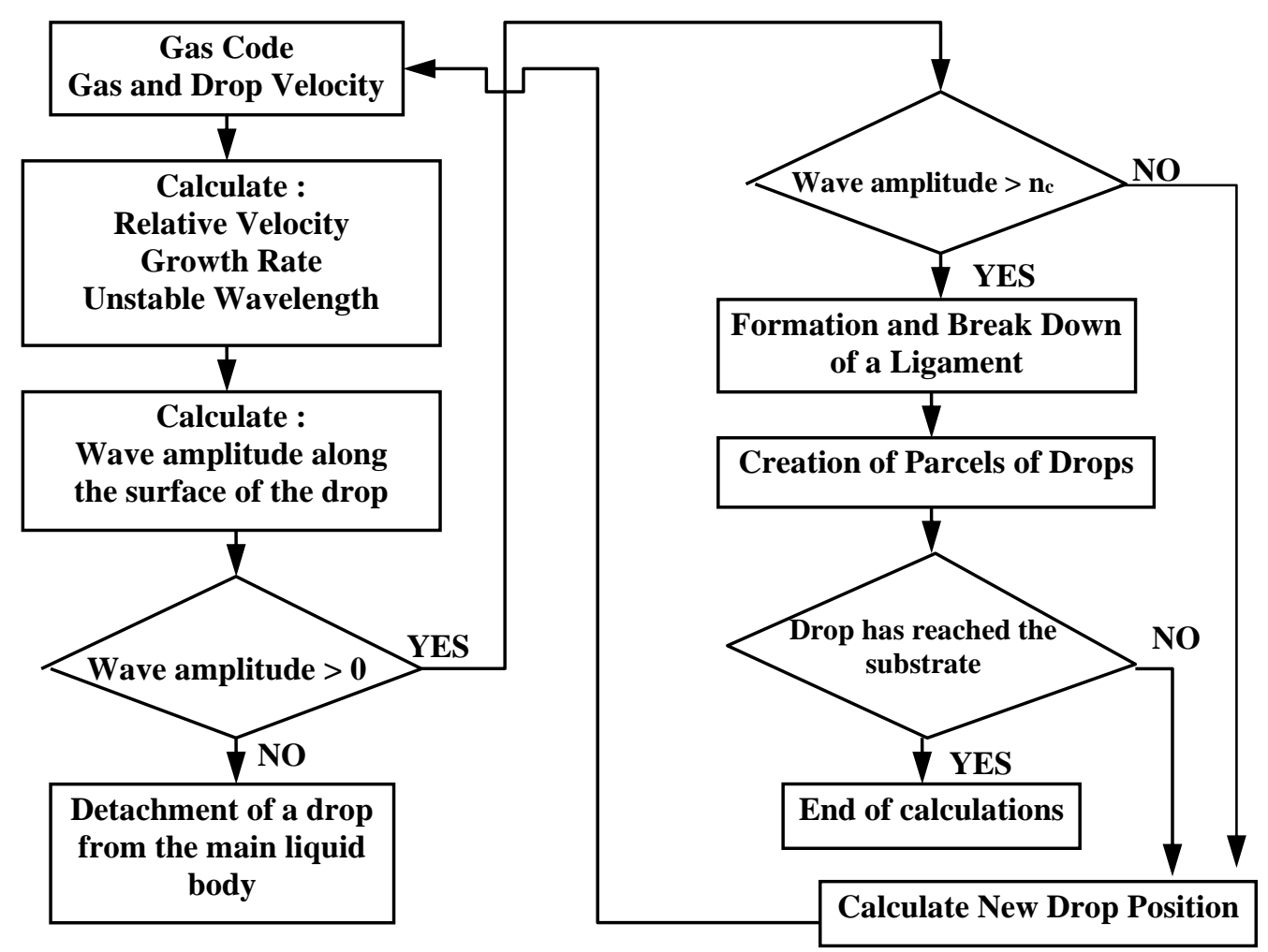

Figure 6 


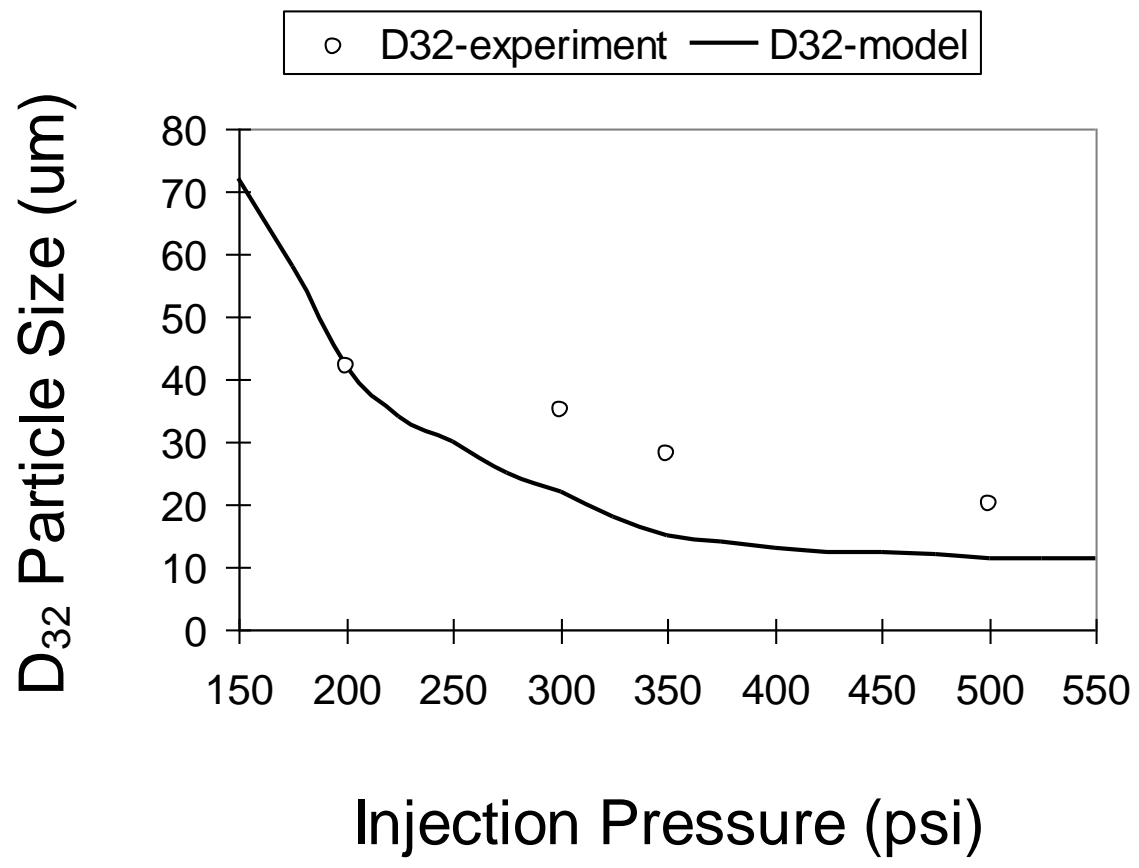

Figure 7

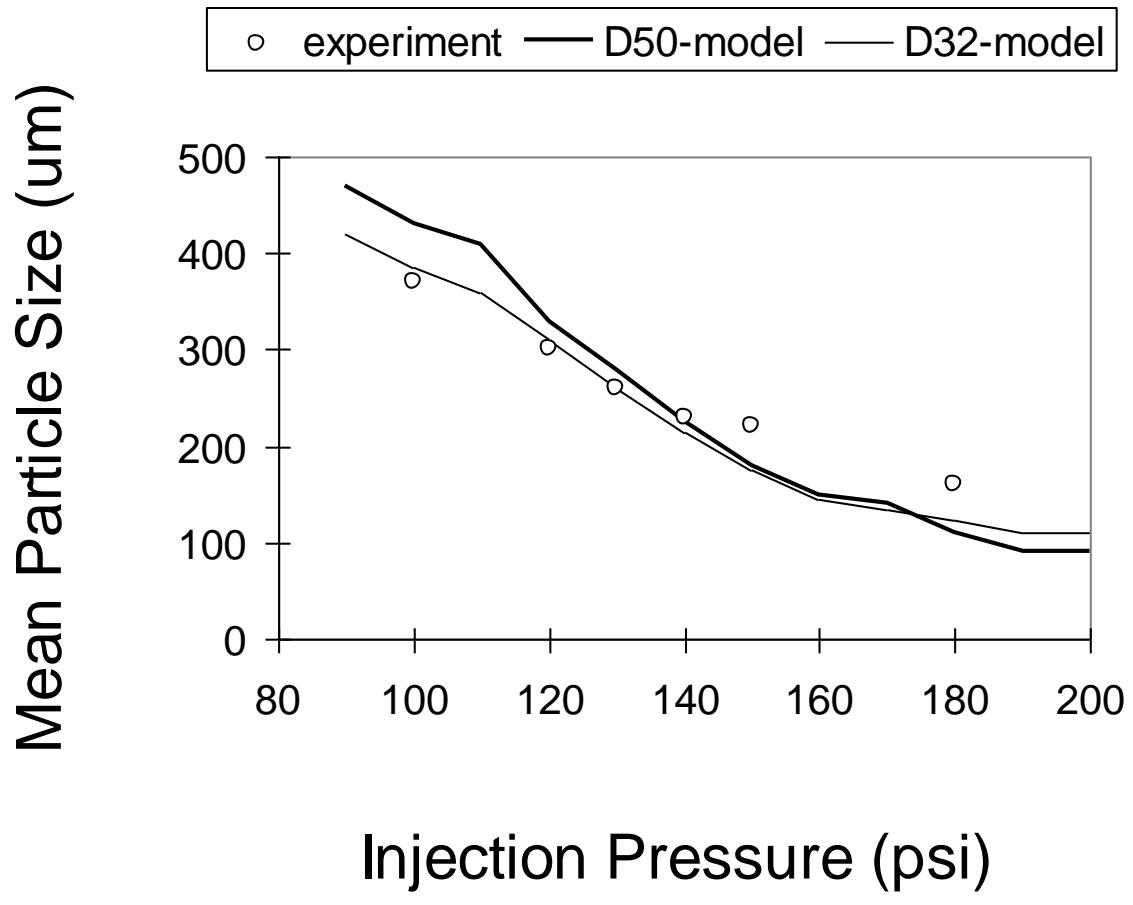

Figure 8 


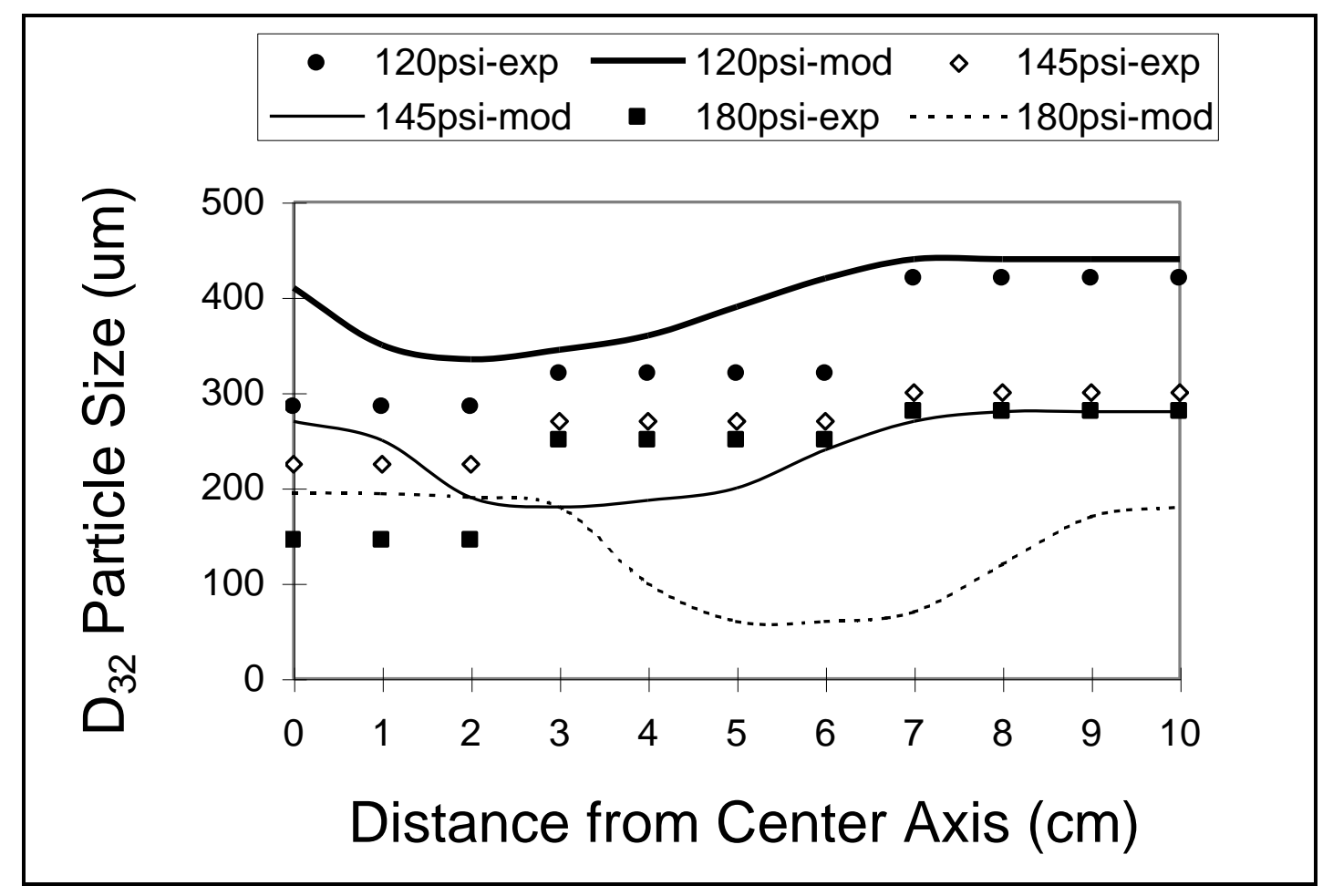

Figure 9

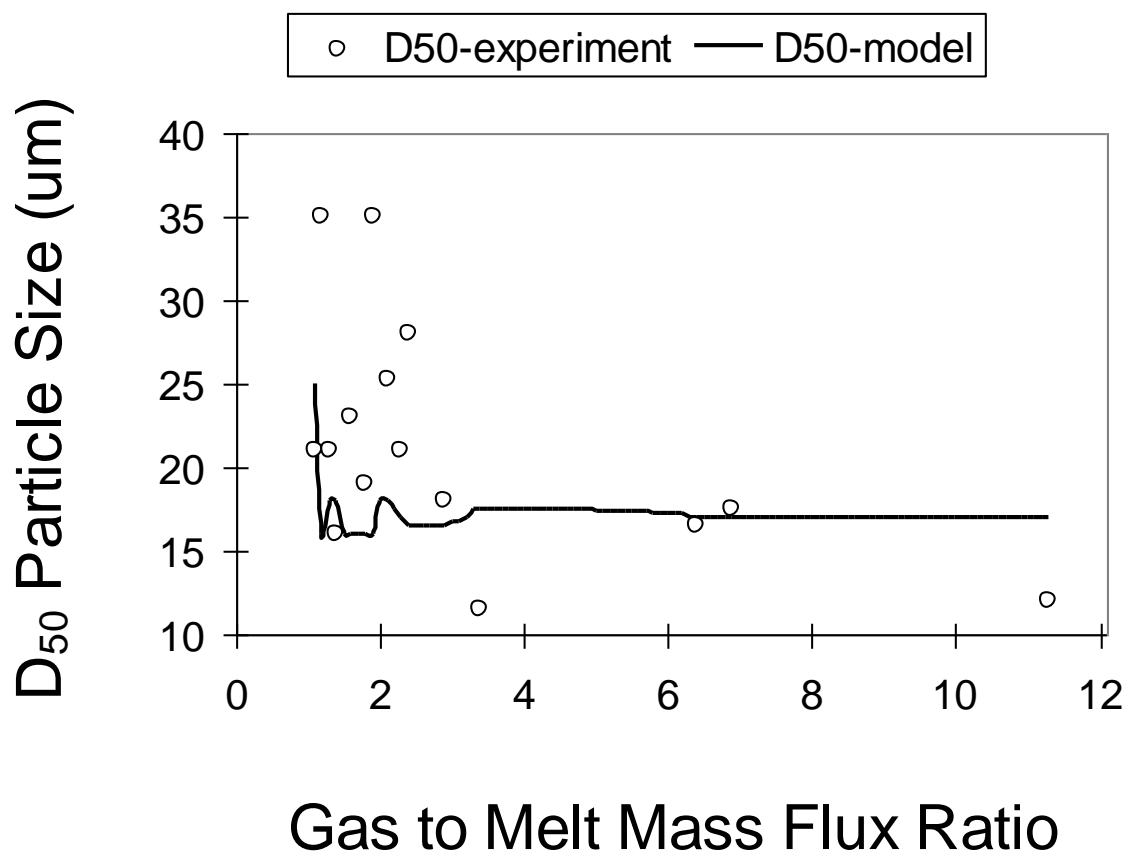

Figure 10 


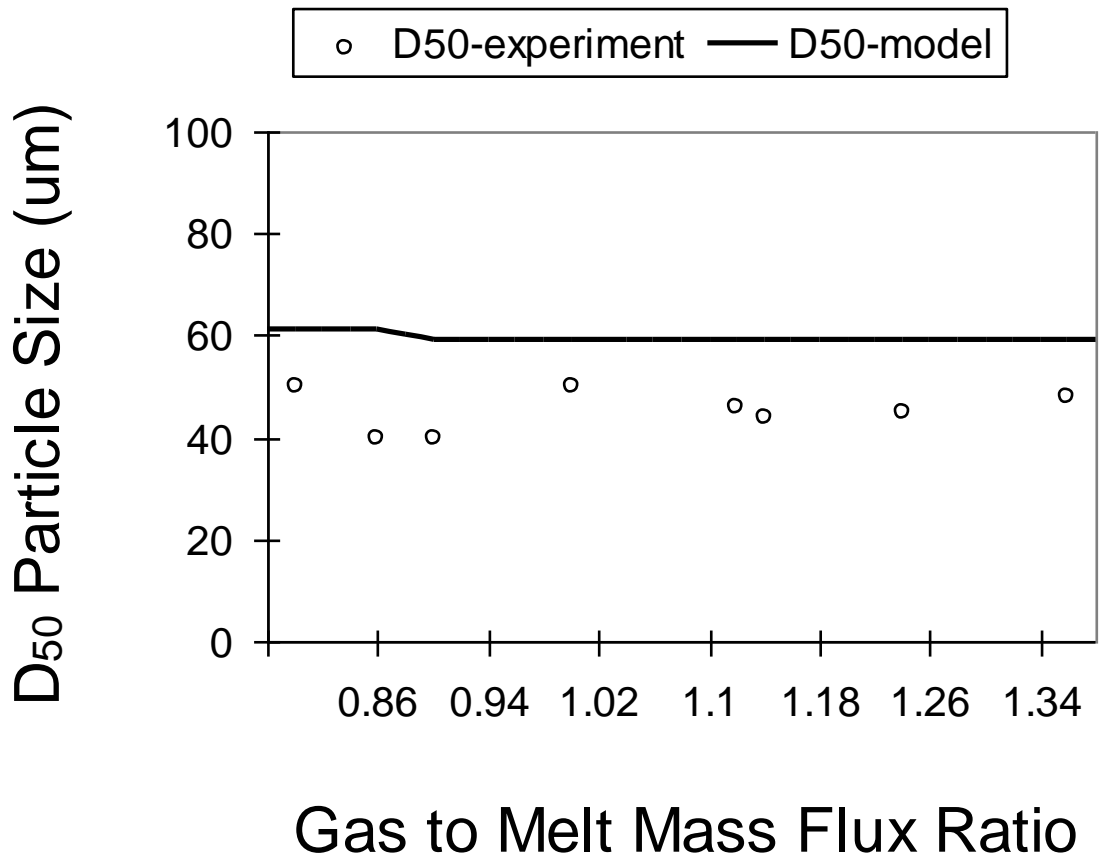

Figure 11 